\title{
Inversión Extranjera Directa de China en América Latina y el Caribe, aspectos metodológicos y tendencias durante 2001-2016
}

Foreign Direct Investment of China in Latin America and the Caribbean, methodological aspects and trends during 2001-2016 Samuel Ortiz Velásquez *

\section{Palabras clave}

Sistemas Económicos Comparados, Inversión y Finanzas Públicas, Política de Inversión, Mercados financieros Key words

Comparative Economics Systems, Investment and Finance, Investment Policy, Financial Markets JeL P5, E62, G31, 016
"Profesor asociado C de tiempo completo adscrito al área de Teorías Aplicadas en la División de Estudios Profesionales de la Facultad de Economía de la UNAM. Contacto: samuelov@economia.unam.mx

\section{4}

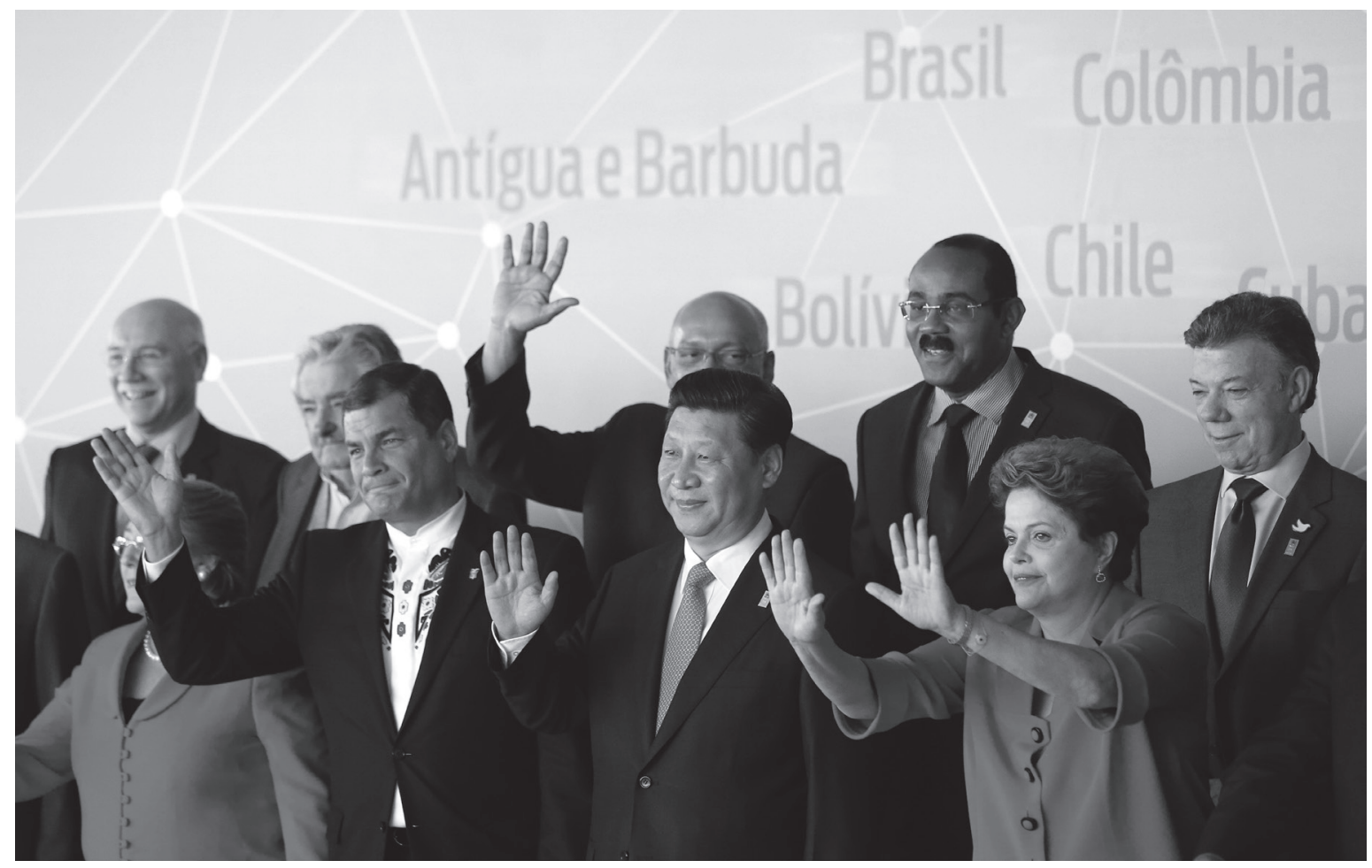

ECONOMÍA INFORMA | 406 | SEPTIEMBRE-OCTUBRE 2017 | 
Samuel Ortiz Velásquez | Inversión Extranjera Directa de China en América

Latina y el Caribe, aspectos metodológicos y tendencias durante 2001-2016

\section{Resumen}

El artículo examina a nivel metodológico y empírico la conducta de la Inversión Extranjera Directa (IED) de China en América Latina y el Caribe (ALC). En la primera parte, se discute el enfoque direccional de la OCDE, pues su implementación es crucial para mejorar las estadísticas mundiales de IED, el enfoque sugiere entre otras cosas, excluir de las estadísticas de IED, la participación de las Entidades con Fines Especiales, pues su inclusión distorsiona el monto, origen y destino de la inversión. La temática es central para China pues usualmente sus empresas canalizan las inversiones por medio de terceros países. El documento destaca la necesidad de distinguir adecuadamente la IED de otros flujos de capital. En la parte empírica, se presenta la metodología y las tendencias de la IED china en ALC para el periodo 20012016, con base en el banco de datos de Dussel Peters y Ortiz Velásquez (2017). Los resultados revelan una alta concentración de la IED china en Brasil, Perú y Argentina, además, destaca que su principal motivación en la región continúa siendo el aseguramiento de materias primas. Así mismo, las empresas chinas han elevado su presencia en la región adquiriendo o fusionándose con empresas locales de mayor tamaño, con ello su contribución a la ampliación del acervo de capital y a la generación empleo es limitada. Desde 2010 está creciendo la participación de la IED china en infraestructura, de continuar dicha tendencia, China podría contribuir a reducir los desequilibrios comerciales que presenta con la región, también podría contribuir a elevar el bajo coeficiente regional de inversión en infraestructura.

\section{Abstract}

The article studies, at the methodological and empirical level, the behavior of China's FDI in Latin America and the Caribbean (LAC). In the first part, the OECD directional approach is discussed, because its implementation is crucial for improving global FDI statistics. The approach suggests, among other things, the exclusion of Special Purpose Entities from FDI statistics, since their inclusion distorts the amount, origin and destination of the investment. The issue is crucial to China, because its companies usually direct their investments through third countries. The paper highlights the need to adequately distinguish FDI from other capital flows. In the empirical part, the methodology and trends of Chinese FDI in LAC are presented for the period 2001-2016, based on the database of Dussel Peters and Ortiz Velásquez (2017). The results show a high concentration of Chinese FDI in Brazil, Peru and Argentina. The main motivation of Chinese FDI continues to be the securing of raw materials. In addition, Chinese companies have increased their presence in the region through by acquiring or merging with large local enterprises, therefore their contribution to the expansion of the capital stock and employment is limited. Since 2010, China's FDI in infrastructure has grown, if this trend continues, China could contribute to reduce the regional trade imbalances and it could contribute to raise the low regional infrastructure investment ratio.

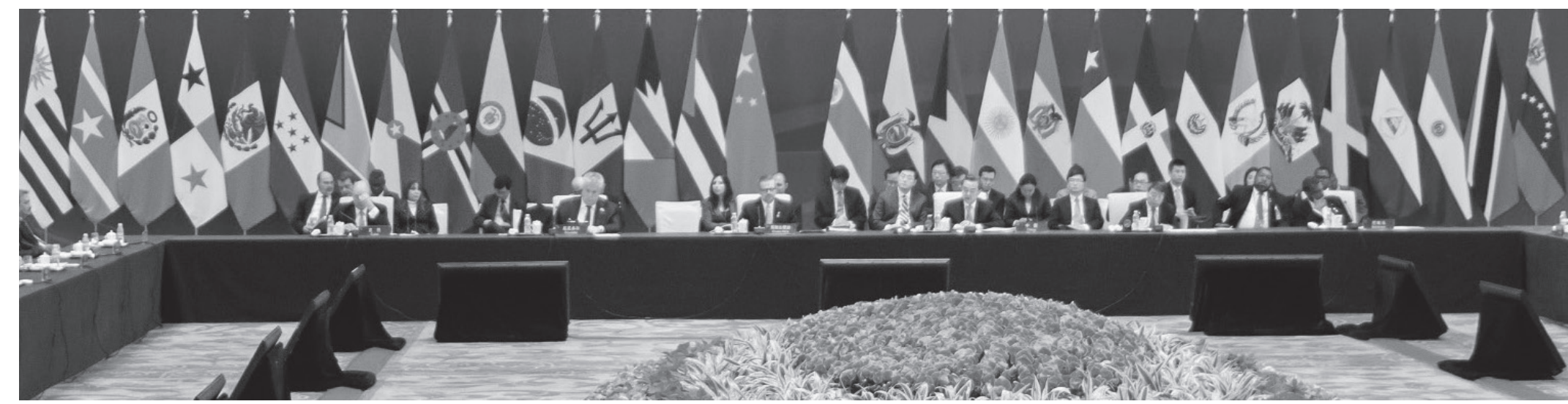




\section{Introducción}

La República Popular China (China en adelante), ha elevado de manera masiva su presencia en el mundo y en la región de América Latina y el Caribe (ALC) desde la década de 2000 y con énfasis desde 2009-2010, a través de salidas de capital que adoptan la forma de proyectos de infraestructura, financiamiento e Inversión Extranjera Directa (IED). Tres antecedentes son relevantes para comprender tal dinámica: el lanzamiento de la política go global en 1999; la publicación del Documento sobre la Política de China hacia América Latina y el Caribe editado por el Ministerio de Comercio de la República Popular China (Mofcom) en 2008, que señala la importancia de AlC en términos de proveedora de recursos vitales para el desarrollo doméstico chino y su relevancia política en miras a un mundo de aspecto multipolar (Stanley y Fernández 2016); el proyecto de inversiones en infraestructura comúnmente conocido como "La Franja y la Ruta" de 2013, el cual fue planteado por el presidente $\mathrm{Xi}$ Jinping en mayo de 2017.

En tal contexto se inscribe la conducta de la IED china en el mundo, la que se ha elevado sistemáticamente desde los 12261 millones de dólares (mdd) en 2005 a más de 170 mil mdd en 2016, convirtiendo a China en la segunda fuente de IED en el mundo (después de Estados Unidos) y en una exportadora neta de capital. Concentrados en ALC, diversas fuentes oficiales apuntan a que la IED china en la región ha crecido significativamente desde 2010 , no obstante, dichas fuentes difieren significativamente en los montos que reportan. Por ejemplo, CEPAL (2016) estima un monto de 6955 mdd durante 2015 y el Ministerio de Comercio de la República Popular China (Mofcom) apunta a un monto de 12610 mdd, casi el doble en comparación con la primera fuente. Las divergencias por país de destino también son altas, mientras Mofcom (2017) apunta que las Islas Vírgenes e Islas Caimán capturaron el 86.35\% de las inversiones chinas en ALC entre 20102015, CePal (2015 y 2016) y Pérez (2017) señalan que Brasil y Perú recibieron cerca del 75\% de la IED china.

El objetivo del documento consiste en ofrecer un examen de la metodología actual usada para registrar la IED en el mundo, al tiempo que se sugiere su reemplazo por otra que capture adecuadamente los flujos de IED. Ello permite de paso aclarar una confusión recurrente en torno a los flujos de capital que adoptan la forma de proyectos de infraestructura, financiamiento e IED. La temática es relevante para discutir la relación de China con ALC, pues la mejora en la calidad de sus estadísticas, es un paso inevitable para comprender la emergencia, las motivaciones y los efectos de la IED china en el desarrollo económico de la región. Para cumplir con tales propósitos, el documento se estructura en cuatro apartados. En la primera parte se discute las metodologías internacionales para registrar la IED en el mundo, confrontando a nivel conceptual y empírico el enfoque activo/pasivo con el direccional. En la segunda se explora el caso de China, contrastando a nivel conceptual y empírico la estadística oficial actual de las salidas de IED, con las recomendaciones de OCDE y con estimaciones recientes de Cepal (2016), Pérez (2017) y Dussel Peters y Ortiz Velásquez (2017). En aras de contribuir a la mejora en la calidad de las estadísticas de la IED china, en la tercera parte se describe sinópticamente la metodología del banco de datos de Dussel Peters y Ortiz Velásquez, al tiempo que se presentan las principales tendencias de la IED china en ALC entre 2001 y 2016. La última parte se dedica a las conclusiones. 


\section{L}

a inversión extranjera directa se puede presentar de dos maneras: estándar, para datos agregados recopilados de acuerdo al principio activo pasivo y; complementaria, para datos desagregados compilados de acuerdo al principio direccional

\section{Inversión Extranjera Directa: aspectos metodológicos}

La inversión extranjera directa se registra actualmente con cargo a dos enfoques metodológicos: el convencional o activo pasivo, para la inversión agregada y un nuevo enfoque, el direccional, para la inversión desagregada (OCDE 2008a). A su vez, la IED se puede presentar de dos maneras: estándar, para datos agregados recopilados de acuerdo al principio activo pasivo $\mathrm{y}$; complementaria, para datos desagregados compilados de acuerdo al principio direccional. Los datos de inversión compilados de acuerdo con el enfoque activo pasivo presentan los siguientes rasgos (OCDE 2008a):

i. Son datos agregados consistentes con el sistema de contabilidad nacional ( $\mathrm{SCN}$ ).

ii. Se basan en los informes de una muestra o de un censo de las empresas de una economía. iii. Se clasifican de acuerdo a la primera contrapartida conocida sin tener en cuenta la naturaleza de las empresas, ni la dirección de la influencia o del control entre las empresas.

$i v$. Incluyen todos los fondos que se canalizan por medio de Entidades con Fines Especiales (EFEs) y el capital distribuido por medio de subsidiarias operativas de empresas transnacionales por cuenta de las compañías matrices. Según
OCDE (2008), las EFEs son entidades legales que tienen pocos (o ningún) empleados y poca (o ninguna) presencia física en la jurisdicción en la que son establecidas por su empresa matriz, la cual usualmente se ubica en una jurisdicción distinta. A menudo se utilizan como instrumentos para recaudar capital o mantener activos o pasivos y no realizan actividades productivas significativas. Ofrecen ventajas fiscales, normativas y de confidencialidad. Su existencia, a menudo se relaciona con los centros financieros establecidos en paraísos fiscales.

v. $\mathrm{Al}$ incluir los fondos transitorios canalizados por EFEs residentes, se asiste a un aumento artificial de los datos por país. Además, si los fondos se canalizan a través de EFEs no residentes u otro tipo de subsidiarias, se distorsiona el origen $\mathrm{y}$ destino de las inversiones.

En contraste, la compilación de estadísticas de inversión con base en el enfoque direccional proporciona una mejor medida de las inversiones extranjeras directas, al separar el monto que involucra fondos que pasan transitoriamente a través de una economía (OCDE 2008a). Efectivamente, la asignación al sector o al país de contrapartida se realiza considerando la contrapartida inmediata en la cadena de inversión (en la economía declarante y en el exterior), pero excluyendo a las EFEs residentes. Además, OCDE (2014) enumera las diferencias más significativas entre uno y otro enfoque:

i. En el enfoque activo/pasivo, los activos incluyen tanto los activos de las matrices resientes como los activos de las filiales residentes; los pasivos incluyen tanto los pasivos de las matrices residentes como los pasivos de las filiales residentes. En el enfoque direccional, las salidas de IED únicamente considera las posiciones de las matrices residentes, mientras las entradas de IED sólo considera las posiciones de las filiales residentes.

ii. Una diferencia sustancial se localiza en el tratamiento que se le da a las inversiones mutuas 


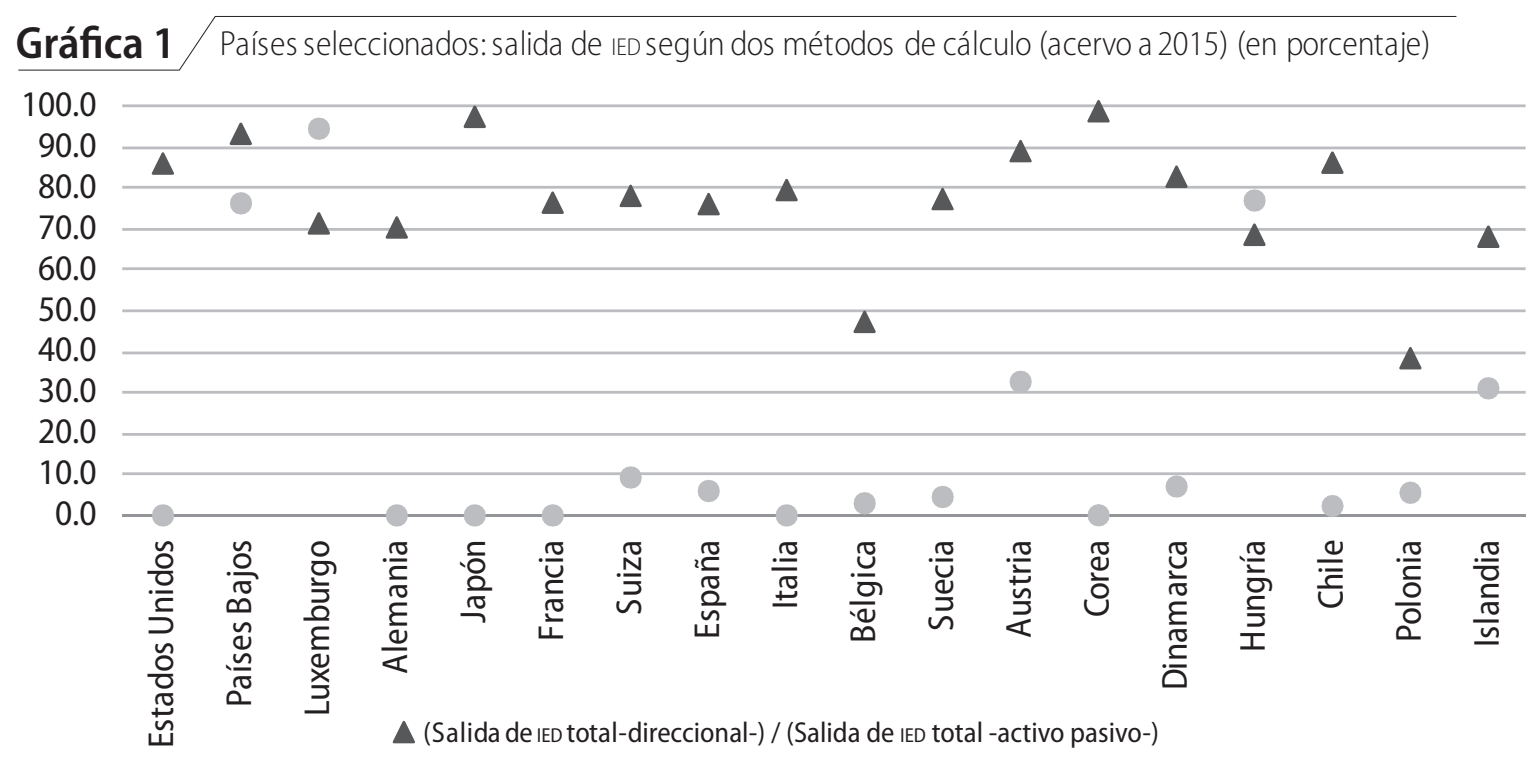

Fuente: elaboración propia con base en OCDE (2017).

o cruzadas (reverse investment). Las inversiones mutuas ocurren cuando una filial invierte en su empresa matriz. Bajo el enfoque direccional la inversión mutua es sustraída para obtener el monto de inversión total en el país declarante.

La OCDE (2017) ha hecho un esfuerzo para estimar estadísticas de inversión extranjera directa separando la actividad de las EFes. En éste contexto, apenas 14 economías han reportado al organismo sus estadísticas de flujos y acervo de IED excluyendo a las EFEs residentes: Austria, Hungría, Luxemburgo, Países Bajos, Chile, Dinamarca, Islandia, Noruega, Polonia, Portugal, España, Suiza, Suecia y el Reino Unido.

$\mathrm{Al}$ respecto la gráfica 1 muestra información de acervo de salidas de IED para una muestra de países a 2015, según el enfoque activo pasivo y el direccional, asimismo, distingue la participación de las EFEs residentes. Varios aspectos son relevantes: i) con la excepción de Japón y Corea, la salida de IED calculada con base en el enfoque direccional es inferior al cálculo según el enfoque activo pasivo, la magnitud del desnivel depende del tamaño y la dirección de las inversiones cruzadas, no obstante, como ha demostrado ocDE (2014), ambas medidas se han movido pari passu en más de un $80 \%$ entre 2012-2013; ${ }^{1}$ ii) con base en el enfoque direccional, las EFEs residentes no son significativas en Estados Unidos, Alemania, Japón, Francia, Italia y Corea; iii) los cuatro países con las mayores participaciones relativas de las EFEs residentes en la salida de IED total, son coincidentemente los países que han venido reportando los datos de forma consistente desde 2005: Luxemburgo (94.61\%), Países Bajos (76.35\%), Hungría (77.05\%) y Austria (32.69\%). Ello revela la importancia de mejorar la cobertura de las estadísticas por país.

Por otro lado, ocDE (2008a) ha sugerido a las economías la compilación y publicación de estadísticas complementarias elaboradas con cargo al enfoque direccional. Las series

1 Ello porque la inversión de las matrices residentes en sus filiales extranjeras suele ser el componente más importante de la salida de IED (enfoque direccional) y de la adquisición de activos (enfoque activo pasivo). 
complementarias son relevantes pues entre otras cosas, permiten clasificar a las inversiones en dos tipos: fusiones y adquisiciones (FyA) e inversiones nuevas (greenfield investments). La distinción es crucial, pues v.gr., las FyA implican únicamente un cambio de propiedad de una empresa previamente establecida, con ello no genera efectos positivos por lo menos en el corto plazo en términos de ampliación del acervo de capital y generación de empleo. De hecho, siguiendo el modelo de crecimiento Harrod-Domar, sólo la IED que adopta la forma de nueva inversión, puede contribuir a elevar el coeficiente de inversión y con ello estimular el crecimiento económico (véase evidencia empírica internacional en Harms y Méon 2011).

La UNCTAD a través del Informe de las Inversiones en el Mundo, es al parecer la única institución internacional que presenta datos agregados de transacciones tipo FyA e inversiones nuevas. En ambos casos, se incluyen transacciones que no están calificadas como IED, según las recomendaciones internacionales (véase Ortiz Velásquez 2016). No obstante, se trata de un primer esfuerzo a nivel internacional en ésta materia.

\section{El caso de las estadísticas de Inversión Extranjera Directa de China}

El gobierno de China ha emprendido desde 2003, diversos esfuerzos por adoptar e implementar los estándares internacionales del FMI y de la OCDE en la recopilación y publicación de estadísticas de IED (OCDE 2008b), no obstante, persiste en la estadística oficial una significativa desviación en sus volúmenes de inversión (cuadro 1). Por ejemplo, según UNCTAD (2017), en 2016 la IED china ascendió a 183, 100 mdd, 16\% menos que la estadística de OCDE y $8 \%$ más que la estadística de Mofcom para ese mismo año. Ello se explica entre otras cosas, porque el Mofcom recopila datos con base en la aprobación y los registros récord de proyectos de IED, por lo cual, los proyectos de inversión emprendidos por empresas que han escapado a los procedimientos de aprobación y registro a través de ésta institución pueden ser omitidos en algunos casos.

Por otra parte, Mofcom en coordinación con la Oficina Nacional de Estadística y la Administración Estatal de Divisa Extranjera, trabajan desde 2014 en ajustes a las estadísticas de la IED china, ello con base en las recomendaciones

Cuadro 1 China: flujo de salida de IED total y a ALC (2005-2015) (millones de dólares)

\begin{tabular}{lcccccccc}
\hline & 2005 & 2010 & 2011 & 2012 & 2013 & 2014 & 2015 & 2016 \\
\hline OCDE & 13730 & 57954 & 48421 & 64963 & 72971 & 123130 & 174391 & 217203 \\
UNCTAD & 12261 & 68811 & 74654 & 87804 & 107844 & 123120 & 127560 & 183100 \\
MofCom & 12261 & 68811 & 74654 & 87804 & 107844 & 123120 & 145667 & 170110 \\
\hline \multicolumn{7}{c}{ Destino: mundo } \\
\hline MofCom & 6466 & 10538 & 11936 & 6170 & 14359 & 10547 & 12610 & $\ldots$ \\
CEPAL & $\ldots$ & 13712 & 10174 & 9206 & 5770 & 10915 & 6955 & $\ldots$ \\
\hline
\end{tabular}

Fuente: elaboración propia con base en OCDE (2017), UNCTAD (2017), Mofcom (2016) y CEPAL (2016). 
más recientes de la oCDE (Mofcom 2015). En suma, pese a los esfuerzos emprendidos por las instituciones chinas, la estadística oficial sigue sin capturar adecuadamente la real magnitud de las salidas de IED de China, particularmente por la práctica recurrente de sus empresas de canalizar la mayor parte de sus inversiones a través de terceros países. ${ }^{2}$

A lo anterior se agregan dos problemas. Primero, desde un enfoque de balanza de pagos, no todo financiamiento externo con participación de empresas extranjeras califica como IED. La clave radica en observar la nacionalidad de la empresa inversora, citemos dos ejemplos:

1. Como señala Cepal (2016), el proyecto del megaresort Baha Mar en las Bahamas casi concluido para 2015, involucró una inversión estimada de 3500 mdd. Pero como el proyecto fue gestado por empresarios locales que contrataron a la empresa China Construction of America, no puede ser considerado como IED. Solo en caso de que el proyecto fuera adquirido por una empresa extranjera, calificaría como IED.

2. En mayo de 2015 el Industrial and Commercial Bank of China (ІСвС) concedió un préstamo de 2000 mdd a Petrobras en Brasil para la compra de equipamiento marino (Expansión 2015). La transacción no califica como IED sino como inversión fija bruta de nacionales, pues la empresa demandante de inversión es nacional. En breve, la transacción figurará en la cuenta de capital del SCN de dos formas: como crédito que mostrará el financiamiento de la inversión y como débito que mostrará la formación de capital real.

2 Por ejemplo, la compra de Sinopec del $40 \%$ de Repsol en el Brasil en 2010, por 7000 mdd, se registró como una inversión de Luxemburgo por haberse canalizado a través de la filial china en dicho país (CEPAL 2015).

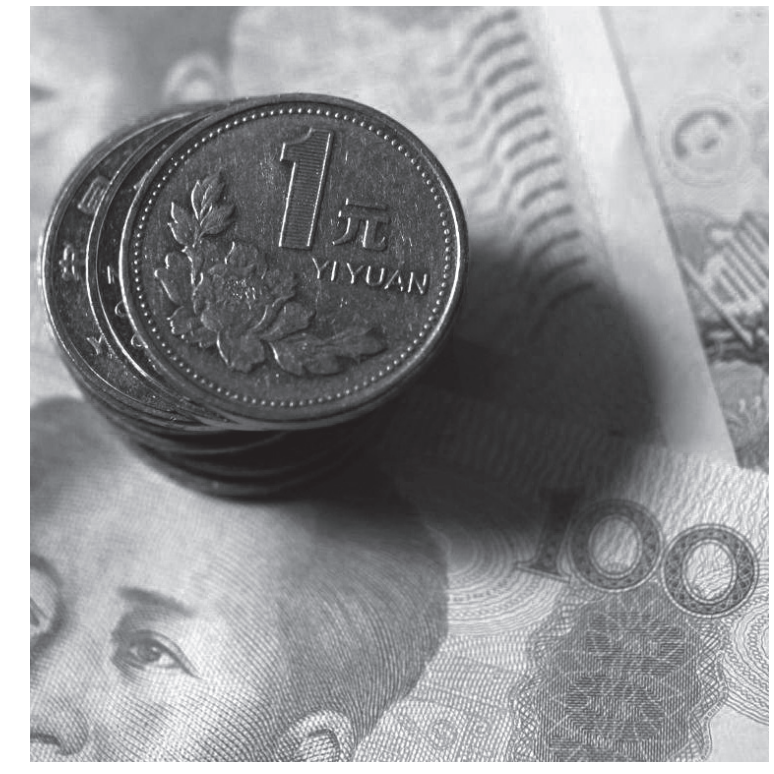

Segundo, algunas economías de ALC receptoras de IED no mantienen registros sobre el origen de las inversiones, v.gr., tres de las mayores 20 transacciones tipo FyA durante 2014, fueron realizadas por empresas chinas. Sin embargo, dos de ellas se produjeron en Perú, cuyo Banco Central no rastrea el origen de las inversiones (CEPAL 2016).

Los argumentos anteriores explican los esfuerzos de Cepal (2015 y 2016), Pérez (2017) y Dussel Peters y Ortiz Velásquez (2016 y 2017), por estimar montos propios de IED china en la región. Incluso el esfuerzo de CEPAL es parcial pues aborda el periodo 2010-2015 e incluye únicamente a 10 economías. En el apartado siguiente se presentan los principales resultados del banco de datos a nivel de empresa de la IED de China en América Latina y el Caribe de Dussel Peters y Ortiz Velásquez (2017), que complementa los esfuerzos realizados por CePal y Pérez (2017). El banco de datos busca contribuir a mejorar la calidad de las estadísticas de la IED china, además, incorpora una primera aproximación al empleo generado por dichas inversiones y ello es un valor agregado con respectos a otros esfuerzos 
empíricos. El banco partió de una recopilación de más de 600 transacciones a nivel de empresa de fDi Markets, Thomson-Reuters, Bloomberg, Capital IQ, China Global Investment Tracker (CGIT) y anuncios de inversión de la prensa especializada, que se complementó con un minucioso análisis de gabinete, con el propósito de destilar las transacciones realizadas que califican como IED considerando los aspectos tratados en los apartados uno y dos. El resultado fue la integración de una serie para 274 transacciones realizadas durante 2001-2016. La base de datos presenta además otras características: $i$ ) cubre a 22 países de la región (Argentina, Barbados, Bermuda, Bolivia, Brasil, Chile, Colombia, Costa Rica, Cuba, Ecuador, El Salvador, Guyana, Honduras, Jamaica, México, Nicaragua, Panamá, Perú, Surinam, Trinidad y Tobago, Uruguay y Venezuela); ii) clasifica a las inversiones en nuevas inversiones y fusiones y adquisiciones; iii) distingue las transacciones por tipo de propiedad de la empresa inversora (pública o privada); iv) clasifica el destino de las inversiones en cuatro tipos de actividad: materias primas, manufactura, servicios y mercado interno y compra de tecnología. ${ }^{3}$

\section{Características de la IED china en ALC: 2001-2016}

Desde 2010 el flujo promedio de IED a la región de ALC ha sido próximo a los 172000 mdd (UNCTAD 2017), dos veces más que el flujo promedio recibido entre 2001-2009. Pero con una caída acumulada de $26.6 \%$ entre 2011 y 2016. En éste contexto la IED china estimada pasó de un monto promedio anual de 1357 mdd

3 La metodología detallada se puede consultar en Dussel Peters y Ortiz Velásquez (2017). La base de datos se puede descargar de: http://www.redalc-china.org/ monitor/informacion-por-pais/busqueda-por-pais entre 2001-2009 a 10817 mdd promedio en 2010-2016, destaca que la IED china alcanzó su tercer mayor nivel en 2016 cuando se situó en 9799 mdd, sólo superada por los más de 23 mil mdd que ALC recibió en 2010 y los más de 18600 mdd de 2014. En ese lapso, la participación ponderada relativa de la IED china como parte de la IED regional pasa de 1.67 a 6.30 por ciento.

El examen a nivel de empresa muestra que durante 2001-2016 las empresas chinas realizaron 274 transacciones con los países de ALC, con un flujo acumulado de 87 mil 928 mdd. El empleo estimado asociado a las inversiones fue de 202 mil 202 empleos, con ello cada empleo fue generado por 434855 dólares (ver cuadro 2). El periodo 2010-2016 atestiguó el máximo dinamismo por el momento, acumulando casi 76000 mdd y 168 mil 806 empleos. No obstante que durante 2001-2016, alrededor de 7 de cada 10 transacciones se vincularon a procesos de ampliación de capital, las nuevas inversiones explicaron apenas $36.46 \%$ de la IED y $49.85 \%$ del empleo. En contraste, las fusiones y adquisidores presentaron un monto promedio por transacción cinco veces superior al que reportaron las nuevas inversiones y representaron más de dos terceras partes de la IED china total.

Las tendencias anteriores son significativas, pues muestran que las empresas chinas han optado en términos generales por adquirir empresas en ALC de gran tamaño, antes que emprender nuevos proyectos de inversión, dicho fenómeno se profundiza en los años que siguieron a la crisis internacional de 2008-2009. De hecho, los años pico en captación de IED china (2010, 2014 y 2016) se han explicado en más de $70 \%$ por las transacciones tipo FyA. Todo lo cual ha tenido implicaciones en términos de generación de empleo, v.gr., entre 2015 
Cuadro 2 ALC: IED China y generación de empleo (2001-2016)

\begin{tabular}{|c|c|c|c|c|c|c|}
\hline Periodo & $\begin{array}{l}\text { Transacciones } \\
\text { (número) }\end{array}$ & $\begin{array}{c}\text { IED } \\
\text { (mdd) }\end{array}$ & $\begin{array}{c}\text { Empleo } \\
\text { (número de traba- } \\
\text { jadores) }\end{array}$ & $\begin{array}{l}\text { IED/Transacción } \\
\text { (mdd) }\end{array}$ & $\begin{array}{l}\text { IED/Empleo } \\
\text { (coeficiente) }\end{array}$ & $\begin{array}{c}\text { Empleo/Transacción } \\
\text { (número de trabaja- } \\
\text { dores) }\end{array}$ \\
\hline \multicolumn{7}{|c|}{ Total } \\
\hline 2001-2009 & 63 & 12210 & 33394 & 194 & 0.366 & 530 \\
\hline $2010-2016$ & 211 & 75718 & 168806 & 359 & 0.449 & 800 \\
\hline 2001-2016 & 274 & 87928 & 202200 & 321 & 0.435 & 738 \\
\hline 2015 & 32 & 3629 & 26300 & 113 & 0.138 & 822 \\
\hline 2016 & 27 & 9799 & 28290 & 363 & 0.346 & 1048 \\
\hline \multicolumn{7}{|c|}{ Nuevas inversiones } \\
\hline 2001-2009 & 47 & 9687 & 21416 & 206 & 0.452 & 456 \\
\hline 2010-2016 & 151 & 22372 & 79372 & 148 & 0.282 & 526 \\
\hline 2001-2016 & 198 & 32059 & 100788 & 162 & 0.318 & 509 \\
\hline 2015 & 26 & 2913 & 4132 & 112 & 0.705 & 159 \\
\hline 2016 & 14 & 1122 & 4465 & 80 & 0.251 & 319 \\
\hline \multicolumn{7}{|c|}{ Fusiones y adquisiciones } \\
\hline 2001-2009 & 16 & 2522 & 11978 & 158 & 0.211 & 749 \\
\hline 2010-2016 & 60 & 53346 & 89434 & 889 & 0.596 & 1491 \\
\hline $2001-2016$ & 76 & 55869 & 101412 & 735 & 0.551 & 1334 \\
\hline 2015 & 6 & 715 & 22168 & 119 & 0.032 & 3695 \\
\hline 2016 & 13 & 8677 & 23825 & 667 & 0.364 & 1833 \\
\hline \multicolumn{7}{|c|}{ Propiedad de la empresa inversora: pública } \\
\hline 2001-2009 & 34 & 10095 & 18894 & 297 & 0.534 & 556 \\
\hline 2010-2016 & 97 & 61081 & 99976 & 630 & 0.611 & 1031 \\
\hline $2001-2016$ & 131 & 71176 & 118870 & 543 & 0.599 & 907 \\
\hline 2015 & 8 & 884 & 932 & 111 & 0.949 & 117 \\
\hline 2016 & 18 & 9066 & 25644 & 504 & 0.354 & 1425 \\
\hline
\end{tabular}

Fuente: elaboración propia con base en Monitor de la OfDI de China en ALC (2017).

y 2016 las nuevas inversiones explicaron apenas cerca del $16 \%$ del empleo generado por la IED china total.

El cuadro 2 también destaca que son las empresas públicas las que más han invertido y generado empleo en la región: entre 20012016 las empresas chinas públicas invirtieron más de 71 mil mdd representando $80.95 \%$ del total y generaron 58.79\% del empleo. En 2016 las empresas públicas chinas elevaron a más de
90\% su participación relativa en las inversiones y empleo. El resultado es relevante desde una perspectiva teórica y empírica, pues dado que es la empresa pública china la que invierte masivamente en ALC, sus motivaciones van más allá del criterio de la rentabilidad privada, bien podría sostenerse que son las estrategias de desarrollo de corto, mediano y largo plazo del gobierno chino, las que están detrás del boom de las inversiones chinas. 
Samuel Ortiz Velásquez | Inversión Extranjera Directa de China en América

Latina y el Caribe, aspectos metodológicos y tendencias durante 2001-2016

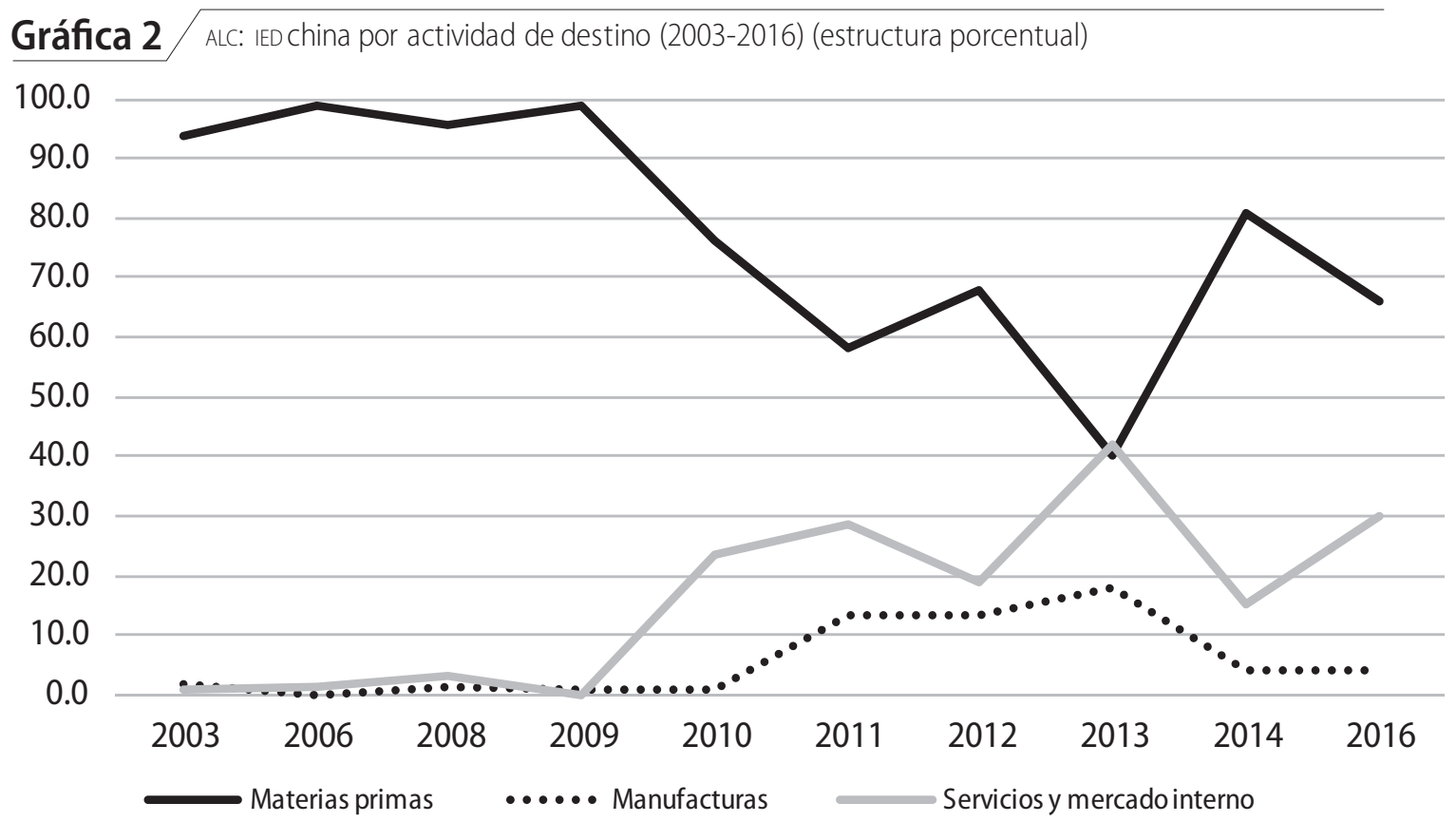

Fuente: elaboración propia con base en Monitor de la ofDI de China en ALC (2017).

Las transacciones chinas en ALC se han concentrado en tres actividades de destino desde 2001: materias primas; manufacturas y; servicios y mercado interno, pero el monto de inversión orientado a materias primas representó casi $71 \%$ y generó $48 \%$ del empleo total (Monitor de la IED de China en ALC 2017). Destaca que el monto promedio por transacción y el tamaño promedio de planta en las transacciones de materias primas duplica en valor al monto promedio total. Es decir, la principal motivación de las empresas chinas en ALC ha sido grosso modo el aseguramiento de materias primas y ello contrasta con la conducta de la IED del resto del mundo, que v.gr., para 2010-2014 se dirigió en apenas $25 \%$ a materias primas (CEPAL 2016) (ver gráfica 2).

No obstante, desde 2009 se asiste a una reorientación en la estructura de la IED china en ALC: de materias primas a las actividades de servicios e infraestructura. Si entre 2003-2009 más de 95\% de la IED china se dirigía a materias primas, desde 2010 ser- vicios ha representado en promedio más de $26 \%$ del total, pasando de participaciones relativas de $1 \%$ durante la década de 2000 a 29\% en 2016 (ver gráfica 2). Tal contra tendencia puede ser relevante para la región, considerando que actualmente ALC invierte en promedio menos de $3 \%$ del PIB en infraestructura (México menos de 2\%), por lo cual la IED china en infraestructura bien puede contribuir a cerrar brechas (Serebrisky, Suárez, et.al. 2015).

En contraste con la estadística de Mofcom, las inversiones chinas en ALC se han concentrado mayoritariamente en Brasil, Perú y Argentina; éstas economías han elevado su participación relativa de 30\% durante 20012009 a $77.85 \%$ en $2010-2016$ (ver cuadro 3). Además, han capturado más de $60 \%$ del empleo generado por la IED. Llama la atención el caso de México, pues si bien capturó apenas $2.28 \%$ de la IED china en ALC durante 20012016, dicha inversión le permitió a México capturar $7 \%$ del empleo regional total. 
Cuadro 3 ALC: IED China por país de destino (2001-2016)

\begin{tabular}{|c|c|c|c|c|c|c|c|c|c|c|}
\hline & $\begin{array}{l}2001- \\
2009\end{array}$ & $\begin{array}{l}2010- \\
2016\end{array}$ & 2015 & 2016 & $\begin{array}{l}2001- \\
2016\end{array}$ & $\begin{array}{l}2001- \\
2009\end{array}$ & $\begin{array}{l}2010- \\
2016\end{array}$ & 2015 & 2016 & $\begin{array}{l}2001- \\
2016\end{array}$ \\
\hline & \multicolumn{5}{|c|}{ OFDI (millones de dólares) } & \multicolumn{5}{|c|}{ Empleos generados (número de empleados) } \\
\hline Total & 12209.57 & 75718.20 & 3628.55 & 9798.86 & 87927.76 & 33394 & 168806 & 26300 & 28290 & 202200 \\
\hline Brasil & 573.7 & 39114.45 & 1822.05 & 8718.76 & 39688.14 & 8674 & 88976 & 14805 & 20201 & 97650 \\
\hline Perú & 3071.4 & 12751.6 & 0 & 0 & 15823 & 8120 & 9815 & 0 & 0 & 17935 \\
\hline Argentina & 3.8 & 7083.2 & 0 & 214.8 & 7087 & 200 & 7118 & 0 & 670 & 7318 \\
\hline Venezuela & 175 & 3937.2 & 278 & 0 & 4112.2 & 848 & 7835 & 40 & 0 & 8683 \\
\hline Chile & 2488.5 & 817.1 & 286 & 212.4 & 3305.6 & 328 & 4685 & 175 & 4267 & 5013 \\
\hline Ecuador & 2377 & 674.74 & 0 & 30.8 & 3051.74 & 2143 & 22561 & 0 & 250 & 24704 \\
\hline México & 1043 & 964.26 & 626.3 & 0.2 & 2007.26 & 7616 & 6722 & 3660 & 0 & 14338 \\
\hline Colombia & 730 & 1221.7 & 0 & 23.9 & 1951.7 & 554 & 1231 & 0 & 275 & 1785 \\
\hline Guyana & 1390.07 & 95.6 & 0 & 0 & 1485.67 & 4442 & 1701 & 0 & 0 & 6143 \\
\hline Resto & 357.1 & 9058.35 & 616.2 & 598 & 9415.45 & 469 & 18162 & 7620 & 2627 & 18631 \\
\hline
\end{tabular}

Fuente: elaboración propia con base en Monitor de la OFDI de China en ALC (2017).

Sumado a la alta concentración por país y actividad de destino, se asiste a una alta concentración agregada de la IED china por empresa. Efectivamente diez empresas chinas, han invertido en ALC alrededor de 54678 mdd entre 2001-2016 (representaron $62.19 \%$ de la IED) y han generado 55233 empleos (representaron $27.32 \%$ del empleo acumulado). Del monto invertido, más de $78 \%$ corresponde a transacciones tipo fusiones y adquisiciones (ver el cuadro 4). Sinopec, MMG Limited, China Three Gorges Corporation, Sinochem, CNPC, State Grid Corporation of China y CNOOC, todas empresas públicas, son de las principales inversoras en ALC. Otro rasgo significativo apunta a que con la excepción de las inversiones de la empresa State Grid Corporation of China cuyas transacciones en Brasil se han orientado a actividades de servicios y mercado interno, el resto de las empresas ha invertido en actividades vinculadas a materias primas. Además, las transacciones más importantes se han concentrado en Brasil, Perú y Argentina. Destaca: la presencia de Sinopec en Brasil que entre 2010 y 2012 adquirió a Repsol por alrededor de 7100 mdd y a Petrogal (Galp), por 4800 mdd, en el mismo país Sinochem adquirió a Statoil por 3100 en 2010; en 2014 la MMG adquirió en Perú el proyecto las Bambas por más de 7000 mdd; en Argentina CNOOC adquiere en 2010 una participación de 50\% de Bridas por $3100 \mathrm{mdd}$. Esto significa que cinco fusiones y adquisiciones vinculadas a materias primas por parte de cuatro empresas públicas, han explicado casi el 30\% de las inversiones chinas en ALC desde 2001.

\section{Conclusiones}

La creciente y abultada exportación de capitales por parte de China al mundo y a la región de ALC desde la década de 2000, que adopta la forma de proyectos de infraestructura, financiamiento e IED, ha motivado un creciente interés en el sector académico por comprender sus motivaciones, conducta y repercusiones socioeconómicas y medioambientales. Concentrando la atención en el monto de IED china, llama la atención las fuertes discrepancias estadísticas existentes según la fuente consultada y el poco 
Samuel Ortiz Velásquez | Inversión Extranjera Directa de China en América

Latina y el Caribe, aspectos metodológicos y tendencias durante 2001-2016

Cuadro 4 ALC: principales 10 empresas chinas inversoras (2001-2016)

\begin{tabular}{|c|c|c|c|c|c|c|}
\hline Empresa & $\begin{array}{l}\text { Actividad } \\
\text { predominante } \\
\text { de destino }\end{array}$ & Tipo & $\begin{array}{l}\text { No. de } \\
\text { transac- } \\
\text { ciones }\end{array}$ & IED (mdd) & $\begin{array}{c}\text { Empleo } \\
\text { (número de } \\
\text { empleados) }\end{array}$ & $\begin{array}{l}\text { Proyectos por: tipo, país de } \\
\text { destino y año }\end{array}$ \\
\hline \multirow{3}{*}{$\begin{array}{l}\text { China Petroche- } \\
\text { mical Corp (Si- } \\
\text { nopec) (Pública) }\end{array}$} & \multirow{3}{*}{$\begin{array}{l}\text { Materias } \\
\text { primas }\end{array}$} & Total & 7 & 17819 & 8381 & \multirow{3}{*}{$\begin{array}{l}\text { FyA: Brasil } 2010 \text { (Repsol) y } 2012 \\
\text { (Petrogal), Argentina } 2010 \text { (Occi- } \\
\text { dental), Colombia } 2006 \text { (Omimex). } \\
\text { Nuevas: Venezuela } 2013 \text { (Orinoco), } \\
\text { Ecuador } 2006 \text { (Crudos Pesados), } \\
\text { Brasil } 2006\end{array}$} \\
\hline & & FyA & 4 & 14780 & 6004 & \\
\hline & & Nuevas & 3 & 3039 & 2377 & \\
\hline $\begin{array}{l}\text { MMG Limited } \\
\text { (Pública) }\end{array}$ & $\begin{array}{l}\text { Materias } \\
\text { primas }\end{array}$ & FyA & 1 & 7000 & 1786 & $\begin{array}{l}\text { FyA: Perú } 2014 \text { (Glencore, Las Bam- } \\
\text { bas Copper Deposit) }\end{array}$ \\
\hline $\begin{array}{l}\text { China Three } \\
\text { Gorges Corp } \\
\text { (Pública) }\end{array}$ & $\begin{array}{l}\text { Materias } \\
\text { primas }\end{array}$ & FyA & 2 & 5400 & 681 & $\begin{array}{l}\text { FyA: Brasil } 2016 \text { (Hydroelectric Uti- } \\
\text { lities Jupia and Ilha Solteira y Duke } \\
\text { Energy International) }\end{array}$ \\
\hline \multirow{3}{*}{$\begin{array}{l}\text { Sinochem Group } \\
\text { (Pública) }\end{array}$} & \multirow{3}{*}{$\begin{array}{l}\text { Materias } \\
\text { primas }\end{array}$} & Total & 5 & 4655 & 1035 & \multirow{3}{*}{$\begin{array}{l}\text { FyA: Brasil } 2010 \text { (Statoil), Colombia } \\
2009 \text { (Emerald Energy), Ecuador } \\
2003 \text { (Ecuador Block } 16 \text { Oilfield). } \\
\text { Nuevas: Colombia 2012, Jamaica } \\
\text { (2011) }\end{array}$} \\
\hline & & FyA & 3 & 3500 & 650 & \\
\hline & & Nuevas & 2 & 1155 & 385 & \\
\hline \multirow{3}{*}{$\begin{array}{l}\text { China National } \\
\text { Petroleum Cor- } \\
\text { poration (CNPC) } \\
\text { (Pública) }\end{array}$} & \multirow{3}{*}{$\begin{array}{l}\text { Materias } \\
\text { primas }\end{array}$} & Total & 6 & 4359 & 22841 & \multirow{3}{*}{$\begin{array}{l}\text { FyA:Perú } 2014 \text { (Petrobras). Nuevas: } \\
\text { Venezuela (2), Ecuador (2), Brasil (1) }\end{array}$} \\
\hline & & FyA & 1 & 2600 & 146 & \\
\hline & & Nuevas & 5 & 1759 & 22695 & \\
\hline $\begin{array}{l}\text { State Grid } \\
\text { Corporation of } \\
\text { China (Pública) }\end{array}$ & $\begin{array}{l}\text { Servicios y } \\
\text { mdo interno }\end{array}$ & FyA & 2 & 4026 & 9634 & $\begin{array}{l}\text { FyA: Brasil } 2016 \text { (CPFL Energia SA) y } \\
2010 \text { (Elecnor, Concesionaria Serra } \\
\text { Paracatu) }\end{array}$ \\
\hline \multirow{3}{*}{$\begin{array}{l}\text { China National } \\
\text { Offshore Oil } \\
\text { Corporation } \\
\text { (CNOOC) (Pú- } \\
\text { blica) }\end{array}$} & \multirow{3}{*}{$\begin{array}{l}\text { Materias } \\
\text { primas }\end{array}$} & Total & 4 & 3870 & 4490 & \multirow{3}{*}{$\begin{array}{l}\text { FyA: Argentina } 2010 \text { (Bridas, Pan } \\
\text { American Energy). Nuevas inversio- } \\
\text { nes: Argentna (2), Trinidad y Tobago } \\
\text { (1) }\end{array}$} \\
\hline & & FyA & 1 & 3100 & 1700 & \\
\hline & & Nuevas & 3 & 770 & 2790 & \\
\hline $\begin{array}{l}\text { Xinfa Group } \\
\text { (Privada) }\end{array}$ & $\begin{array}{l}\text { Materias } \\
\text { primas }\end{array}$ & Nuevas & 1 & 3000 & 159 & Nuevas: Jamaica 2014 \\
\hline \multirow{3}{*}{$\begin{array}{l}\text { Aluminium } \\
\text { Corporation of } \\
\text { China (Chinalco) } \\
\text { (Pública) } \\
\end{array}$} & \multirow{3}{*}{$\begin{array}{l}\text { Materias } \\
\text { primas }\end{array}$} & Total & 3 & 2559 & 4144 & \multirow{3}{*}{$\begin{array}{l}\text { FyA: Perú } 2013 \text { (Pomacocha Power). } \\
\text { Nuevas: Perú } 2008 \text { y } 2009\end{array}$} \\
\hline & & FyA & 2 & 385 & 1055 & \\
\hline & & Nuevas & 1 & 2174 & 3089 & \\
\hline \multirow{3}{*}{$\begin{array}{l}\text { CITIC Group } \\
\text { Corp (Pública) }\end{array}$} & \multirow{3}{*}{$\begin{array}{l}\text { Materias } \\
\text { primas }\end{array}$} & Total & 2 & 1990 & 2082 & \multirow{3}{*}{$\begin{array}{l}\text { FyA: Brasil } 2011 \text { (Moreira Salles } \\
\text { family, Companhia Brasileira de } \\
\text { Metalurgia y Mineração). Nuevas: } \\
\text { Venezuela } 2014\end{array}$} \\
\hline & & FyA & 1 & 1950 & 1800 & \\
\hline & & Nuevas & 1 & 40 & 282 & \\
\hline \multirow{3}{*}{$\begin{array}{l}\text { Subtotal prime- } \\
\text { ras } 10\end{array}$} & \multirow{3}{*}{$\begin{array}{l}\text { Predomina } \\
\text { materias } \\
\text { primas }\end{array}$} & Total & 33 & 54678 & 55233 & \multirow{3}{*}{$\begin{array}{l}\text { FyA: Brasil } 2011 \text { (Moreira Salles } \\
\text { family, Companhia Brasileira de } \\
\text { Metalurgia y Mineração). Nuevas: } \\
\text { Venezuela } 2014\end{array}$} \\
\hline & & FyA & 17 & 42741 & 23456 & \\
\hline & & Nuevas & 16 & 11937 & 31777 & \\
\hline
\end{tabular}

Fuente: elaboración propia con base en Monitor de la OFDI de China en ALC (2017). 
trabajo empírico efectuado para superar tales debilidades. Considerando que la mejora de las estadísticas, es un paso inevitable para el trabajo empírico y para formular recomendaciones de política económica realistas en la relación económica de China con ALC, el documento exploró y contrastó diversas metodologías utilizadas para registrar los flujos de IED total y la realizada por China. Posteriormente se presentó un examen de la metodología y las tendencias de la IED china en la región con base en el banco de datos elaborado por Dussel Peters y Ortiz Velásquez (2017), que busca contribuir a solventar las debilidades metodológicas observadas.

En el ámbito metodológico es crucial para las naciones (no solo para China), avanzar en la adopción del enfoque direccional de la OCDE para cuantificar la IED, así como en la elaboración de cuentas complementarias que permitan clasificar la inversión en nuevas inversiones y fusiones y adquisiciones. La temática es central, pues como se deriva del análisis empírico, la creciente presencia de IED china particularmente en Brasil, Perú y Argentina, a través de fusiones y adquisiciones, no está contribuyendo a ampliar el acervo productivo de la región ni a generar nuevos empleos, al tiempo que significa la entrada de nuevos actores chinos con capacidad de incidir en el funcionamiento de las economías.

Es relevante que en el trabajo empírico se distinga adecuadamente los flujos de IED de otros que adoptan la forma de financiamiento y proyectos de infraestructura, pues como se discutió en el documento, no todo financiamiento externo con participación de empresas extranjeras califica como IED. Incluso los proveedores privados de datos usualmente utilizados en el análisis empírico de la IED como Thomson-Reuters, fDi Markets, et.al., incurren comúnmente en éstos errores. La distinción de la participación de las Entidades con Fines Especiales residentes y no residentes en los flujos de IED en coherencia con el enfoque direccional, es otro tema metodológico a mejorar, considerando que usualmente las empresas chinas canalizan sus inversiones por medio de terceros países, distorsionando con ello el monto, origen y destino de las salidas de IED. Las economías latinoamericanas también tienen que mejorar la contabilidad de la IED v.gr., Perú no clasifica la IED según el origen.

En la parte empírica se presentaron las principales tendencias de la IED china en ALC desde 2001, con base en Dussel Peters y Ortiz Velásquez (2017). En contra de la estadística oficial de Mofcom (que apunta a que más del $80 \%$ de la IED china se ha concentrado en Islas Vírgenes e Islas caimán), el análisis mostró que el máximo dinamismo del flujo de inversión china desde 2010 se explica en lo básico por la presencia de empresas trasnacionales chinas que han concentrado sus esfuerzos en Brasil, Perú y Argentina. Apenas diez multinacionales chinas públicas han elevado su presencia en la región vía fusiones y adquisiciones en los sectores petroleros y minería, sin efectos en el corto plazo en ampliación del acervo de capital de la región. Por otro lado, la creciente presencia en actividades de servicios y mercado interno, parece vincularse estrechamente con el proyecto de la Franja y la Ruta, de continuar ésta tendencia, la IED china puede tener el potencial de contribuir a reducir el creciente comercio asimétrico y a cerrar brechas de desarrollo vía su aporte en elevar el bajo coeficiente regional de inversión en infraestructura. Los resultados presentados son una invitación a profundizar en la comprensión de la IED china en ALC a nivel de empresa. ? 
Samuel Ortiz Velásquez | Inversión Extranjera Directa de China en América Latina y el Caribe, aspectos metodológicos y tendencias durante 2001-2016

\section{Bibliografía}

Cepal. 2015. América Latina y el Caribe y China. Hacia una nueva era de cooperación económica. CEPAL, Santiago de Chile.

CEPAL. 2016. Relaciones económicas entre América Latina y el Caribe y China. Oportunidades y Desafios. CEPAL, Santiago de Chile, noviembre.

Dussel Peters, Enrique y Samuel Ortiz Velásquez. 2016. Monitor de la OFDI de China en México año 1, número 1, marzo. Red Académica de América Latina y el Caribe sobre China, México.

Dussel Peters y Ortiz Velásquez. 2017. Monitor de la OFDI de China en América Latina y el Caribe. Red Académica de América Latina y el Caribe sobre China, México.

Expansión. 2015. "Petrobras obtiene un préstamo de 2.000 millones del banco chino ІсвC". Expansión, octubre 13

Harms, Philipp y Pierre-Guillaume Méon. 2011. An FDI is an FDI is an FDI? The growth effects of greenfield investment and mergers and acquisitions in developing countries. Proceedings of the German Development Economics Conference, Berlin 2011, No. 38

International Monetary Fund (imF). 2015. Coordinated Direct Investment Survey (CDIS). Proyect on Bilateral Asymmetries. DMSDR1S-\#5436883-v2Paper, june 13.

Ministry of Commerce of the People's Republic of China (Mofcom). 2017. [http://fec.mofcom.gov.cn/].

Monitor de la ofdi de China en América Latina y el Caribe. 2017. Estadísticas. Información por país. [http://www.redalc-china.org/monitor/informacion-por-pais/busqueda-por-pais]. Fecha de consulta: 12.08.2017.
OCDE. 2008. OCDE Benchmark Definition of Foreign Direct Investment. Fourth edition. OCDE, Paris OECD. 2008b. OECD Investment Policy Reviews: China 2008. oecD.Duss

OECD. 2014. Implementing the latest international standards for compiling foreign direct investment statistics. Assetlliability versus directional presentation. OCDE (diciembre).

OECD. 2017. FDI statistics according to Benchmark Deffnition 4th Edition (BMD4). [http://www.oecd.org/ investment/statistics.htm]. Consultado el 3 de julio de 2017.

Ortiz Velásquez, Samuel. 2016. Monitor de la IED de China en América Latina y el Caribe. Aspectos Metodológicos (2000-2016). Red ALC-China, México.

Pérez Ludueña, Miguel. 2017. Chinese Investments in Latin America Opportunities for growth and diversification. Series Production Development 208. CEPAL, Santiago de Chile, abril.

Serebrisky, Tomás, Ancor Suárez-Alemán, Diego Margot, Maria Cecilia Ramirez. 2015. Financiamiento de la infraestructura en América Latina y el Caribe: ¿Cómo, cuánto y quién? Inter-American Development Bank (IDB), Washington DC.

Stanley, Leonardo y José Fernández Alonso. 2016. "El tratamiento a las inversiones extranjeras tras el ascenso de la República Popular China: ¿̇de las reglas a la discreción?". Cuadernos de Trabajo del Cechimex, número 3,16 págs.

UnCTAD. 2017. World Investment Report 2017. Investment and the digital economy. UnCTAD, Geneva. 